\title{
Fabrication of Patient Specific Genioplasty Surgical Template Using Investment Casting
}

\author{
Santosh Kumar Malyala ${ }^{1^{*}}$, Ravi Kumar $\mathbf{Y}^{1}$, Rakesh $\mathrm{K}^{2}$ and Chitra Chakravarthy ${ }^{3}$ \\ ${ }^{1}$ Department of Mechanical Eengineering, National Institute of Technology, Warangal, Telangana, India \\ ${ }^{2}$ Department of Mechanical Engineering, Bheema Institute of Technology and Science, A.P, India \\ ${ }^{3}$ Department of Oral and Maxillo Facial Surgery, Navodaya Dental College Raichur, Karnataka, India
}

*Corresponding author: Santosh Kumar Malyala, Department of Mechanical Engineering, National Institute of Technology Warangal, Telangana-506004, India, Tel: +91 9963343361; E-mail: msantoshpdd@gmail.com

Received date: June 01, 2017; Accepted date: July 19, 2016; Published date: July 26, 2017

Copyright: ( 2017 Malyala SK, et al. This is an open-access article distributed under the terms of the Creative Commons Attribution License, which permits unrestricted use, distribution and reproduction in any medium, provided the original author and source are credited.

\begin{abstract}
Additive Manufacturing ( $A M)$ is one of the best techniques to fabricate customized medical models. This technique best suited for the medical industry. The surgical template design and dimensions vary from patient to patient, depending on the anatomy of the patient. AM provides flexibility to design a patient specific surgical template using the patient CT scan data. Medical processing software converts DICOM to 3D CAD data. This 3D CAD data is used to design the patient specific surgical template. This surgical template data is then converted into an STL file format to fabricate medical models using AM machines. The initial surgical template is fabricated using FDM machines, which are easily available and this template is used for pre surgical planning. After satisfactory results are obtained in the pre surgical planning, the same STL file is fabricated using a castable resin. The castable resin model is used for preparation of the mould for casting process. This mould is then used to produce the final surgical template with medical grade SS316. The cost of the final metal surgical template is reduced by 30 percent compared to production of same model fabricated using metal AM system. The major advantage of this process is that we obtain a patient specific template in traditional approved way, but at reduced cost.
\end{abstract}

Keywords: Additive manufacturing; Computer Aided Design (CAD); Genioplasty; Surgical template; Casting

\section{Introduction}

The 3D printing of medical models has been there for more than 25 years. In the initial, days this technology was named as Rapid Prototyping (RP). As the name indicates these are mainly used as prototypes in many industries one of them being medical. When this technology was introduced in 1980's there were only very few machines across the globe. The cost of these machines was very high in those days and the choice of material was also very limited [1]. As technology progress machines became affordable for common people, especially the machines with Fused Deposition Modelling (FDM) technique. FDM machines have a wide range of materials choice. The beauty of 3D printing technology is that each and every model made of 3D printer can be customized [2]. This is benefiting most of the medical industry. In case of medical industry almost each and every patient requires customized medical models such as surgical templates or implants [3].

The first development in the medical industry took place in 1895 with discovery of X-ray by wilhelm rochene. This helped to analyze the bone anatomy of the patient in those days. The radiograph provides only $2 \mathrm{D}$ anatomy of the patient that too only bony information of the patient. To overcome, this CT scanner was designed by incorporating multiple X-rays around the gantry tube. Depending on the number of emitters and receivers the CT scanner specification varies [4-6]. In the initial days there used to be dual slice CT scanners, Advancement in technology now enable 256 slice CT scan. Generally CT scanners are used to acquire bone anatomy of patient whereas MRI is used to acquire soft tissue information of the patient [7]. In the early stages of AM the models, they were used for pre surgical planning only, but in the current period AM is capable of producing direct implants as per patient specific requirements [8]. Although the technology is so advanced, there still are issues to identifying certified medical implant manufacturers, availability of machine, time and cost of implants or template. These processes still need to be streamlined. To overcome the medical approvals in case of AM medical models an alternate approach has been identified in the current work, which utilizes the maximum benefit of AM technology along with conventional casting process.

Under the AM roof there are several processes comes, classification of which is done based upon the technique or material used. Broadly it can be classified based on the raw material used can whether liquid, powder or solid. The easily available technique among these is the FDM technique, where the solid filament is used as a raw material. Since this technology is easily available and economical, it is used largely in fabricating of pre surgical models for planning. FDM parts have good strength; these models can be used for functional testing and also for the pre surgical planning as in the case of mock surgery. The medical models can be cut according to the surgical plan. Genioplasty is one of such the complex procedures done in oral and maxillofacial surgery. Use of a surgical template makes genioplasty easier. In genioplasty the anterior mandible is cut and moved as per patient requirement. To identify the cutting region of bone using CT or $\mathrm{X}$-ray data is very difficult. The designed template provides highest advantage to mark the cutting region on the mandible and mini plates are also bent in prior to surgery using AM medical model.

The procedure followed for casting of patient specific surgical template is shown in the flowchart below (Figure 1). 


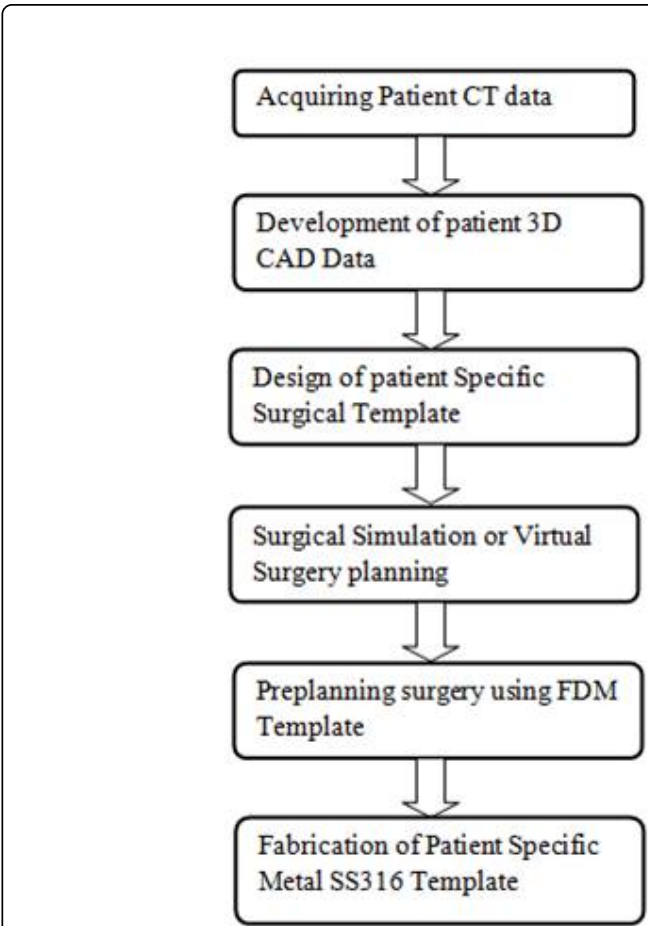

Figure 1: Flow chart of current work.

\section{Acquiring patient $\mathrm{CT}$ data}

The CT data of the patient is acquired using Somatom 128 slice CT scanner with $3 \mathrm{D}$ face scan. The acquired DICOM image is reconstructed using optimal reconstruction parameters. The CT scan data of the patient is shown in the Figure 2 below. The DICOM data of the patient is processed using MIMICS, medical processing software to obtain the 3D CAD bone anatomy of the patient. The 3D CAD data of the patient is shown in the Figure $2 b$ below. From the DICOM data the bone anatomy of the patient is segregated using Hounsfield Units value, In the MIMICS the HU value for the bone starts from 226 to 3071. The soft tissue HU value is below 256 in the MIMICS software.

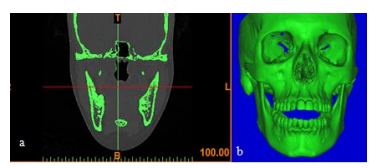

Figure 2: (a) 2D scan data; (b) 3D CAD data of patient.

\section{Development of patient 3D CAD data}

From the complete bony anatomy of the patient the area of interest is seperated using operations like region growing, edit mask etc. In this study for the development of a srgecial template for genioplasty, the anterior mandible is the region of instrest. The 3D CAD mandible of the patient is shown in the Figure 3 below [4]. the 3D CAD data of mandible is used for the development of patient specific template..

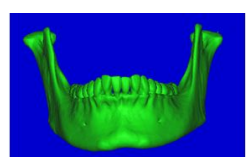

Figure 3: Patient 3D CAD mandible.

\section{Design of patient specific surgical template}

A patient specific surgical template was designed to fit exactly along the contour mandible of the patient, as shown in the Figure 4a below. The purpose of this template is to get perfect symmetry during the bony cut. Also, the cut is expected to be at $30^{\circ}$ angulation to enable this angulation, a $30^{\circ}$ chamfer was created at the edge of the template for the saw or drill to follow this angulation without any deviation. the template was designed in a way to avoid important structure such as the mental nerve on both sides and also the root apices. The 3D CAD image of template with patient mandible is shown in Figure $4 \mathrm{~b}$ below.

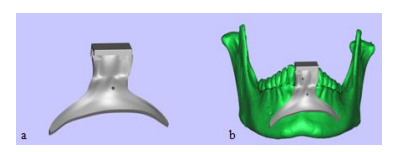

Figure 4: (a)Patient specific surgical template. (b)3D CAD mandible with surgical template.

\section{Surgical simulation or virtual surgery planning}

MIMCS software provides the flexibility of performing a virtual, the bone can be cut and position as planned to analyze the result as shown in the Figure 5. After obtaining the expected results the design of the template is finalized otherwise the iteration will be repeated. Since this is only virtual surgery, the same procedure is conducted using the physical AM medical models. To conduct the preplanning surgery FDM models are best suitable and for the current case study to fabricate AM models, the 3D CAD data of the model is converted in to the STL file format.

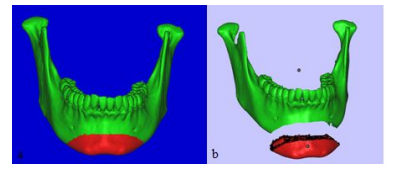

Figure 5: Patient's mandible before virtual surgery. Patient's mandible after virtual surgery.

\section{Pre planning surgery using FDM template}

The STL file format is a neutral file format for all the AM machines. The STL file is processed using preprocessing software to generate a machine specific code to fabricate AM medical model or template. In the current sudy Flashforge finder of FDM technology was used to fabricate the pateient specific medical model and surgical template for preplanning as shown in the Figure 6 below. Flashprint was the preprocessing software for the flashforge finder machine. PLA 
(PolyLacticAcid) was the material used for the fabrication of patient's mandible, mandible osteotomy was done and patient specific surgical template was fabricated. FDM provides the flexibilty of multiple colours of filament to fabricate the model. These models will has good strength, which can be used as functional prototype models also. The biggest advantage of FDM is ease of availability and low cost.

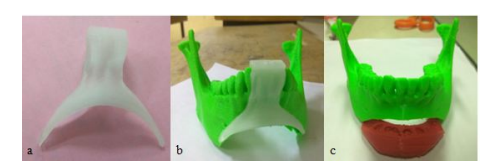

Figure 6: (a) FDM surgical template; (b) FDM mandible with template; (c) Mandible after preplanning surgery.

\section{Fabrication of patient specific metal SS316 template}

For the current study investment casting process was followed to fabricate the patient specific surgical template with SS316 medical grade metal surgical template. The first step to be followed in investment casting is to prepare a pattern which can be evaporated and should be exact replica of the final product. Castable resin pattern of patient specific surgical template for the casting is prepared with SLA process as shown in Figure 7a. This pattern is used to create a mould, which can be used only once, since the mold cannot be opened, to remove the final part mould need to be destroyed.

The advantage of this process is any complex model can be fabricated easily. To create the mould the casting pattern is dipped into slurry of fine grained silica, binder and water. This slurry is formed a ceramic layer over the casting pattern. When the ceramic coating is thick enough as shown in Figure $7 \mathrm{~b}$ it is allowed it to dry. To evaporate the pattern this mould is heated at $150^{\circ} \mathrm{C}$, which forms the cavity for investment casting. The ceramic mould is heated up to $1000^{\circ} \mathrm{C}$ to strengthen the mould and also to drain out any castable resin that is left over. The molten SS316 is poured into the mould, when it is still hot, so that the molten metal will flow easily to each minute feature of the mold. This pouring of metal in to hot mould also results in better dimensional accuracy, since the mold and casting will shrink together as they cool. The final SS316 patient specific surgical template is shown in the Figure $7 \mathrm{c}$ and $7 \mathrm{~d}$, which can be used in surgery. These metal templates can be autoclaved for use during surgery.

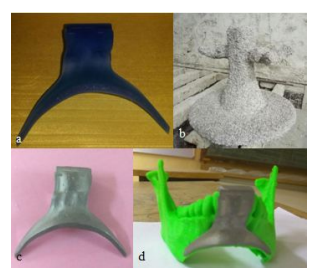

Figure 7: (a) SLA pattern; (b) ceramic mold; (c) SS316 surgical template; (d) metal template and FDM mandible.

\section{Conclusion}

Additive manufacturing technology is best suited for fabrication of customized low volume models. One of the biggest barrier with the metal AM systems is medical grade certification, cost and choice of material, to overcome these barriers a hybrid approach has been followed. The pattern is fabricated with low cost AM systems, which are easily available to everyone. The final required metal surgical template is obtained through traditional investment casting, which is also easily available. The advantage of using this hybrid technique is for the current work SS316 of medical grade is used, but this procedure allows to fabricate model with any material. This procedure provides maximum benefit to the customized parts especially in case of medical industry to fabricate the customized surgical templates and implants.

\section{References}

1. Chua CK, Leong KF (2000) Rapid prototyping. Principles and Applications in Manufacturing. World Scientific.

2. Gibson I, Cheung LK, Chow SP, Cheung WL, Beh SL, et al. (2004) The use of rapid prototyping to assist medical applications. Assises Europeennes de Prototypage Rapide.

3. Krishna LSR, Manmadhachary A, Reddy PB, Venkatesh S (2011) Development of optimum preplanning for maxillofacial surgery using selective laser sintering. International Journal of Engineering, Science and Technology 3: 185-196.

4. Manmadhachary A, Kumar YR, Krishnanand L (2016) Improve the accuracy, surface smoothing and material adaption in STL file for RP medical models. J Manuf Process 21: 46-55.

5. Karatas OH, Toy E (2014) Three dimensional imaging techniques: A literature review. Eur J Dent 8: 132-140.

6. Banerjee P, Rudra S, Ghosh M, Panose P (2013) CT Scans in primary survey for polytrauma patients. Advances in Computed Tomography 2 : 46-51.

7. Malyala SK, Kumar YR (2017) Optimization of computer tomography reconstruction parameters using additive manufacturing. Biol Med (Aligarh) 9: 377 .

8. Malyala SK, Manmadhachary A, Kumar YR, Alwala A (2017) Manufacturing of patient specific AM medical models for complex surgeries. Materials Today: Proceedings 4: 1134-1139. 